\title{
VARIAÇÃO DE MATÉRIA SECA E DE NUTRIENTES NAS FOLHAS E NOS FRUTOS, PRODUÇÃO DE ÁCIDO ASCÓRBICO E SUCO, EM SEIS CULTIVARES DE CITROS, DURANTE UM CICLO
}

\author{
H.P. HAAG (in memoriam); L.E. GUTIERREZ; A.R. DECHEN \\ Departamento de Quimica, ESALQ/USP - C.P. 9 - CEP: 13418-900 -PIRACICABA,SP \\ F.A.A. MOURÃO FILHO; C.S. MOREIRA \\ Departamento de Horticultura, ESALQ/USP - C.P. 9 - CEP: 13418-900 -PIRACICABA,SP
}

RESUMO: De uma plantação de citros, com os cultivares T. Cravo (Cürus reticulata Blanco), L.Hamlin (Cürus sinensis (L.) Osbeck), T. Murcott (Cïrus reticulata Blanco x Citrus sinensis (L.) Osbeck), L. Natal (Citrus sinensis (L.) Osbeck, L. Valencia (Citrus sinensis (L.) Osbeck) e L. Pera (Citrus sinensis (L.) Osbeck), situada na "Fazenda Sete Lagoas", no município de Mogi-Guaçu (22 $2^{\circ}$ 'S, $46^{\circ} 56^{\prime}$ W.Gr.), em Latossolo Vermelho amarelo, fase arenosa, foram coletados frutos 30 dias após florescimento, até a idade da coleta comercial. No material coletado, foram determinadas a variação da matéria seca, a concentração dos macro e micronutrientes nas folhas adjacentes ao fruto, a extração de macro e microuutrientes pelos frutos, a produção de suco $(\mathrm{ml})$ por fruto e a concentração de ácido ascórbico (mg/100 ml de suco). Concluiu-se que: 1.0 aumento da matéria seca, intensifica-se a partir do segundo més apos o florescimento; 2. Com exceção da T. Cravo, ocorre uma diminuição na produção de matéria seca no final do ciclo; 3. A concentração dos macro e micronutrientes nas follhas apresenta oscilações durante o desenvolvimento do fruto; 4 . A ordem decrescente de extração de nutrientes $6: \mathrm{K}, \mathrm{N}, \mathrm{Ca}, \mathrm{Mg}, \mathrm{P}=\mathrm{S}, \mathrm{Fe}, \mathrm{B}, \mathrm{Zn}$, Mn. Cu; 5. A capacidade de exportação de nutrientes pelos cultivares é, em ordem decrescente: L. Pera, L. Hamlin = T. Cravo, T. Murcott, L. Valência, L. Natal; 6. A quantidade de suco produzido por fruto, oscila entre 43 a 95 $\mathrm{ml} ;$ 7. A concentração de ácido ascórbico (mg/100 ml de suco), varia entre 30 a 95.

Descritones: T. Cravo, L. Hamlin, T. Murcott, L. Natal, L. Valência, L. Pera, extração de nutrientes, ácido ascórbico, suco.

\section{SIX CITRUS CULTTVARS COMPARATTVEYY EVALUATED AS TO THEIR FRUIT AND LEAF DRY WEIGHTS AND NUTRIENT CONCENTRATIONS}

SUMMARY: The experiment was carried out in a commercial citrus orchard located in Mogi-Guaçu $\left(22^{\circ} 22^{\prime} S .4^{\circ}\right.$ 56'WGr.), State of São Paulo, Brazil. Five orange types were studied: Cravo (Citrus reticulata Blanco); Hamlin (Citrus sinensis (L.) Osbeck); Natal (Citrus sinensis (L.) Osbeck); Valencia (Citrus sinensis (L.) Osbeck) and Pera (Citrus sinensis (L.) Osbeck) and a tangerine Murcott (Citrus reticulata Blanco x Citrus sinensis (L.) Osbeck). Fruits were picked from 30 days after flowering to the peak of commercial harvest. Leaves nearest the fruits were collected at the same time as the fruits. Sampled material was used to determine: fruit dry weight, macronutrient and micronutrient concentration in the leaves, nutrient extraction by the fruits, juice production per fruit and ascorbic acid concentration in the juice. Fruit dry weight increased following the second month after flowering. Except for Cravo orange fruit dry weight decreased at the end of the cycle. Macronutrient and micronutrient concentrations in the leaves showed variations during fruit development. Rate of nutrient extraction in decreasing order was: $K$, $\mathrm{N}, \mathrm{Ca}, \mathrm{Mg}, \mathrm{P}=\mathrm{S}, \mathrm{Fe}, \mathrm{B}, \mathrm{Zn}, \mathrm{Mn}$ and $\mathrm{Cu}$. The cultivar that exported the largest amount of nutrients was Pera followed by Hamlin = Cravo, Murcott, Valencia and Natal. Juice per fruit ranged between 43 and 95 mililiters whereas ascorbic acid concentration ranged between 30 and 95 miligrams per 100 mililiters of juice.

Key Words: Cravo orange, Hamlin orange, Murcott tangerine, Natal orange, Valencia orange, Pera orange, nutrient extraction, ascorbic acid, orange juice.

\section{INTRODUÇÃO}

\section{Segundo SAMUELS (1957), à} semelhança do cafeeiro, pode-se afirmar que os citros possuem duas "bocas" para alimentar, a primeira para a formação dos frutos para a próxima colheita e a segunda para o novo crescimento da árvore, que é necessária para a formação dos frutos que vão constituir a próxima safra. Dados de extração de nutrientes pelos frutos mais antigos que se conhecem são de Colby e Boschi, citados por CHAPMAN (1968). 
Os autores determinaram as concentrações de $\mathrm{Ca}, \mathrm{Mg}, \mathrm{K}, \mathrm{Na}, \mathrm{P}, \mathrm{S}, \mathrm{Si}$ e $\mathrm{Cl}$ nas cinzas de frutos de cultivares de laranjas e de limões. Observaram que a concentração mais elevada foi de $\mathrm{K}$, seguido de $\mathrm{Ca}$ e a menor foi de Si. Um trabalho muito citado é de LABANAUSKAS \& HANDY (1972), que determinaram as quantidades de nutrientes removidos por frutos de $\mathbf{L}$. Valência (Citrus sinensis (L.) Osbeck). Observaram que os nutrientes exportados em maiores quantidades, foram $\mathrm{K}$ e $\mathrm{N}$ entre os macronutrientes e $\mathrm{Cu}$ e $\mathrm{Mn}$ entre os micronutrientes.

Concluiram os autores que quantidades relativamente pequenas de nutrientes são removidos pela colheita, considerando as quantidades aplicadas ao solo.

No Brasil, os primeiros dados de extração por frutas cítricas são de BATAGLIA et al. (1978). Os autores amostraram na época da colheita, na Estação Experimental de Limeira' do "IAC", frutos dos cultivares "Baianinha", "Hamlin", "Natal", "Valência", "Cravo", "Taiti" e "Murcott". Os frutos foram fracionados em casca, polpa e "suco sementes". Concluiram que as quantidades médias de elementos extraidos em gramas por tonelada de frutos frescos, foram:

$\mathrm{N}-1906 ; \mathrm{P}-173 ; \mathrm{K}-1513 ; \mathrm{Ca}-526 ; \mathrm{Mg}-127 ;$ $\mathrm{S}-137 ; \mathrm{B}-2,2 ; \mathrm{Cl}-24,7 ; \mathrm{Fe}-6,6 ; \mathrm{Mn}-2,8$; Mo-0,008; $\mathrm{Zn}-0,9 ; \mathrm{Co}-0,003 ; \mathrm{Na}-43,5$ e $\mathrm{Al}-7,6$.

Os cultivares com maior capacidade de extração de elementos foram as laranjeiras Natal e Valência e de menor capacidade o limoeiro Taiti.

MALAVOLTA et al. (1988) amostraram mensalmente frutos cítricos "Hamlin", "Natal", "Pera", "Taiti" e "Murcott", na Estação Experimental de Limeira do "IAC", durante um ciclo de produção. Determinaram o diâmetro dos frutos, peso da matéria seca e os teores de micronutrientes. O B e o $\mathrm{Fe}$, foram os nutrientes exportados em maiores quantidades; sendo que o cultivar "Natal" e "Murcott" extrairam maiores quantidades destes nutrientes.

O Cu foi extraido e exportado em menor quantidade. Uma tonelada de frutos frescos exportaram em gramas as seguintes quantidades médias: $\mathrm{B}$ - 3,45; Cu - 0,46; Fe - 4,91; Mn - 0,98; Zn - 1,11.

\footnotetext{
${ }^{1}$ Atual Estação Experimental "Sylvio Moreira"
}

MALAVOLTA et al. (1984) apresentaram dados referentes à variação da matéria seca e de macronutrientes nos frutos de cinco cultivares de citros durante o seu crescimento. Foram amostrados mensalmente, frutos dos cultivares "Hamlin", "Natal", "Pera", "Taiti" e "Murcott",colhidos na Estação Experimental de Limeira do "IAC". Verificaram que o peso da matéria seca intensificou-se a partir do segundo mês após o florescimento. As quantidades médias de nutrientes, exportados pelos cultivares, em $\mathrm{kg}$ por tonelada em frutos frescos, foram: $\mathrm{N}-1,67 ; \mathrm{P}-0,17 ; \mathrm{K}-2,18 ; \mathrm{Ca}-0,66$; $\mathrm{Mg}-0,17 ; \mathrm{S}-0,42$.

O presente trabalho tem por objetivos analisar seis cultivares de citros, com a finalidade de:

- determinar a variação de matéria seca durante um ciclo;

- determinar as concentrações dos macro e micronutrientes nas folhas;

- determinação e extração dos macro e micronutrientes mensalmente, durante o desenvolvimento dos frutos;

- avaliar a produção de suco por fruto nos seis cultivares;

- determinar as quantidades de ácido ascórbico produzidas nos seis cultivares.

\section{MATERIAL E MÉTODOS}

Frutos cítricos, pertencentes aos cultivares Cravo (Citrus reticulata Blanco), Hamlin (Citrus sinensis (L.) Osbeck), Murcott (Citrus reticulata Blanco $\mathrm{x}$ Citrus sinensis (L.) Osbeck) Natal (Citrus sinensis (L.) Osbeck), Valência (Citrus sinensis (L.) Osbeck), Pera (Citrus sinensis (L.) Osbeck), cultivados na "Fazenda Sete Lagoas" no município de Mogi-Guaçu (22 $22^{\circ}$ 'S., $46^{\circ} 56^{\prime}$ W.Gr.), no Estado de São Paulo. Foram coletados mensalmente durante um ciclo. Os cultivares apresentavam idade superior a 20 anos com exceção de $T$. "Murcott" que tinha 7 anos de idade. $O$ porta enxerto, em todos os cultivares, foi limão cravo. Os pomares estão situados sobre Latossolo Vermelho - amarelo, fase arenosa. A adubação empregada foi diferenciada para os diversos cultivares, sendo que os fertilizantes foram 
aplicados, em gramas por pé por ano, nas seguintes quantidades:

$\begin{array}{lrrr}\text { Cultivar } & \mathrm{N} & \mathrm{P}_{2} \mathrm{O}_{5} & \mathrm{~K}_{2} \mathrm{O} \\ \text { T. Cravo } & 350 & 350 & 350 \\ \text { L. Hamlin } & 1260 & 404 & 1620 \\ \text { T. Murcott } & 770 & 335 & 770 \\ \text { L. Natal } & 1115 & 310 & 900 \\ \text { L. Valência } & 1060 & 192 & 1518 \\ \text { L. Pera } & 1215 & 360 & 1440\end{array}$

Os micronutrientes, foram aplicados em gramas por planta, na faixa de: $\mathrm{Zn}=5,4-17,6$; $\mathrm{Mn}=3,4-11,3$ e $\mathrm{B}=1,5$.

Frutos dos cultivares foram coletados a partir dos 30 dias após o florescimento até à maturação comercial, em cerca de vinte por amostragem no início, diminuindo o número à medida que os frutos se desenvolviam, mas nunca inferior a seis frutos por amostragem. Na mesma ocasião folhas adjacentes aos frutos foram coletadas. O material coletado foi lavado, descontaminado e analisado, de acordo com os métodos descritos em SARRUGE \& HAAG (1974). Em amostras avulsas de frutos,foi determinada a quantidade de suco e a concentração de ácido ascórbico pelo método titulométrico do 2,6-diclorofenolindofenol (JACOBS, 1958).

\section{RESULTADOS E DISCUSSÃo}

Crescimento dos frutos.

O crescimento em função das variações do peso da matéria seca nos seis cultivares acha-se exposto na figura 1 . Em todos os cultivares observa-se crescimento acentuado do $1^{\circ}$ ao $3^{\circ}$ mês de idade. $O$ peso da matéria seca é maior no $8^{\circ}$ mês, com exceção da L. Hamlin, em que a máxima produção de matéria seca ocorreu no $4^{\circ}$ mês. A diminuição no peso da matéria seca ao final do ciclo, deve-se à coleta de frutos no interior da árvore (ERICKSON, 1968).

Um fato digno de nota é que todos os frutos dos cultivares, apresentaram peso máximo de matéria seca em torno de 20 a $25 \mathrm{~g}$ por fruto.

\section{Concentração de macronutrientes nas folhas e extração pelos frutos.}

Durante o desenvolvimento dos frutos, ocorreram oscilações acentuadas nas concentrações dos nutrientes nas folhas adjacentes aos frutos como se observa na TABELA 1 , sendo de difícil interpretação, fato este igualmente observado por MALAVOLTA et al. (1984) em L.Taiti, L.Hamlin, T.Murcott, L.Natal e L.Pera.

De modo geral, houve diminuição nas concentrações de $\mathrm{K}, \mathrm{Mg}$ e S em todas as cultivares com o avançar da idade dos frutos. Provavelmente ocorreu transferência desses nutrientes para os frutos. As concentrações de $\mathrm{P}$ e $\mathrm{Ca}$ sofreram pequenas oscilações. A concentração de $\mathrm{N}$ aumentou em T. Cravo e L. Hamlin, apresentando-se nas demais cultivares com oscilações. SMITH (1961) mostra situação semelhante, em folhas coletadas de galhos não frutíferos.

\section{Concentração dos micronutrientes nas folhas e extração pelos frutos.}

Pelo exame da TABELA 2, observa-se que a concentração dos micronutrientes, sofreu oscilações bem menores do que nos macronutrientes. A concentração de B nos seis cultivares é praticamente semelhante, variando de 22 a $63 \mathrm{ppm}$. Para o $\mathrm{Cu}$, a variação foi bem mais ampla, variando de $6 \mathrm{ppm}$ a $291 \mathrm{ppm}$.

Chama atenção, a elevada concentração nas folhas deste micronutriente, em T.Murcott $e$ L.Valência.

A concentração de $\mathrm{Fe}$ é elevada em todas os cultivares, como era esperado. As concentrações de $\mathrm{Mn}$ e $\mathrm{Zn}$ apresentaram-se dentro da faixa considerada como normal e/ou alta (RODRIGUEZ, 1991).

A extração de micronutrientes pelos frutos, de modo geral, foram mais elevados quando confrontados com os dados de MALAVOLTA et al. (1988) para os cultivares Hamlin, Murcott, Natal e Pera, possivelmente devido às práticas de adubação.

Exportação de nutrientes pelos frutos por cultivar.

Pelo exame da TABELA 3 observa-se, inicialmente, uma diferença acentuada na produção de frutos pelos cultivares (FIGUEREDO, 1991).

O elemento exportado em maiores quantidades, em todos os cultivares, foi $\circ \mathrm{K}$, seguido do $\mathrm{N}, \mathrm{Ca}, \mathrm{Mg}, \mathrm{P}$ e finalmente o $\mathrm{S}$. Destaca-se a elevada exportação de $\mathrm{Ca}$, superando inclusive o $\mathrm{K}$ na T. Murcott. 
TABELA 1 - Concentração percentual nas folhas e miligramas dos nutrientes nos frutos em função da idade.

\begin{tabular}{|c|c|c|c|c|c|c|c|c|c|c|c|c|}
\hline \multirow{2}{*}{$\begin{array}{l}\text { Nutri- } \\
\text { entes }\end{array}$} & \multicolumn{12}{|c|}{ Idade dos frutos em meses apos florescimento } \\
\hline & 1 & 2 & 3 & 4 & 5 & 6 & 7 & 8 & 9 & 10 & 11 & 12 \\
\hline & \multicolumn{12}{|c|}{ T. Cravo } \\
\hline $\mathrm{N} \%$ & 1,67 & 1,70 & 1,68 & 1,58 & 3,00 & 1,68 & 2,12 & 2,38 & - & $\cdot$ & - & $\cdot$ \\
\hline N-mg & 14,90 & 39,30 & 51,57 & 156,41 & 104,85 & 106,62 & 111,62 & 166,78 & - & - & • & + \\
\hline $\mathbf{p q}$ & 0,10 & 0,11 & 0,10 & 0,11 & 0,11 & 0,13 & 0,14 & 0,17 & - & - & - & - \\
\hline P-mg & 1.56 & 5,35 & 7,01 & 22,18 & 16,16 & 16,22 & 16,20 & 17,25 & - & - & - & . \\
\hline $\mathbf{K} \%$ & 2,00 & 2,14 & 2,06 & 2,02 & 1,60 & 1,30 & 1,03 & 1,95 & - & - & . & - \\
\hline$K-m g$ & 17,73 & 60,13 & 89,68 & 261,78 & 210,80 & 187,99 & 149,57 & 218,98 & - & - & - & - \\
\hline $\mathrm{Ca} \%$ & 4,70 & 1,56 & 2,83 & 2,66 & 2,77 & 4,23 & 3,63 & 2,77 & - & . & - & - \\
\hline Ca-mg & 3,76 & 15,93 & 57,87 & 95,24 & 115,86 & 229,37 & 67,93 & 223,19 & - & - & •. & - \\
\hline$M_{g} \%$ & 0,53 & 0,33 & 0,22 & 0,50 & 0,29 & 0,36 & 0,35 & 0,37 & . & - & - & - \\
\hline$M g-m g$ & 2,37 & 4,51 & 5,95 & 25,39 & 16,16 & 11,32 & 15,21 & 23,45 & . & - & - & - \\
\hline $5 \%$ & 0,13 & 0,33 & 0,12 & 0,17 & 0,19 & 0,18 & 0,15 & 0,18 & - & - & - & . \\
\hline \multirow[t]{2}{*}{$s-m g$} & 2,05 & 12,03 & 6,08 & 14,75 & 12,28 & 8,42 & 10,23 & 19,49 & - & - & - & - \\
\hline & \multicolumn{12}{|c|}{ L. HAMLIN } \\
\hline $\mathrm{N} \mathscr{F}$ & 2,59 & 2,33 & 2,19 & 2,52 & 2,31 & 2,76 & 3,27 & 3,08 & - & - & - & - \\
\hline$N-m g$ & 35,16 & 75,41 & 122,31 & 101,76 & 138,00 & 150,71 & 176,59 & 190,68 & - & - & - & - \\
\hline $\mathbf{P} \tilde{x}$ & 0,10 & 0,10 & 0,08 & 0,12 & 0,09 & 0,11 & 0,11 & 0,09 & - & - & - & - \\
\hline P-mg & 3,38 & 7,99 & 9,37 & 12,52 & 14,72 & 14,49 & 13,77 & 19,26 & - & - & $\cdot$ & - \\
\hline $\mathrm{K}$ 第 & 1,85 & 1,78 & 1,85 & 1,98 & 1,70 & 1,60 & 1,40 & 1,33 & - & - & - & - \\
\hline$k \cdot m g$ & 45,93 & 112,69 & 145,56 & 172,10 & 211,38 & 203,58 & 116,48 & 238,33 & - & - & - & - \\
\hline $\mathrm{Ca} \%$ & 1,85 & 1,78 & 1,85 & 2,99 & 2,53 & 3,74 & 2,31 & 3,08 & - & - & - & - \\
\hline Ca-mg & 13,39 & 40,81 & 28,09 & 43,08 & 70,61 & 164,84 & 61,47 & 112,66 & - & - & - & - \\
\hline $\mathrm{Mg} \%$ & 0,38 & 0,23 & 0,20 & 0,26 & 0,23 & 0,15 & 0,22 & 0,19 & - & - & - & - \\
\hline $\mathrm{Mg}-\mathrm{mg}$ & 5,15 & 6,93 & 8,40 & 10,31 & 16,39 & 6,04 & 13,36 & 18,33 & - & - & - & - \\
\hline$s \%$ & 0,15 & 0,30 & 0,18 & 0,22 & 0,16 & 0,16 & 0,23 & 0,11 & - & - & - & - \\
\hline \multirow[t]{2}{*}{ S-mg } & 4,33 & 14,09 & 10,18 & 4,96 & 7,85 & 6,78 & 17,93 & 14,71 & - & - & - & - \\
\hline & \multicolumn{12}{|c|}{ T. MURCOTT } \\
\hline $\mathrm{N} \%$ & - & 2,32 & 2,53 & 2,51 & 2,52 & 2,54 & 2,79 & 3,08 & 2,71 & 2,34 & - & - \\
\hline$N-m g$ & - & 45,57 & 126,07 & 219,37 & 133,49 & 198,62 & 196,66 & 230,08 & 237,61 & 187,83 & - & - \\
\hline P\% & - & 0,09 & 0,09 & 0,11 & 0,11 & 0,10 & 0,12 & 0,12 & 0,09 & 0,09 & - & - \\
\hline P-mg & . & 4,01 & 11,35 & 13,11 & 14,11 & 20,46 & 18,98 & 21,76 & 17,75 & 14,14 & - & - \\
\hline$K \%$ & . & 1,57 & 1,56 & 1,43 & 1,60 & 0,63 & 0,75 & 0,94 & 0,82 & 0,92 & - & - \\
\hline $\mathrm{K}-\mathrm{mg}$ & - & 53,39 & 138,33 & 217,51 & 210,06 & 259,77 & 157,40 & 272,22 & 228,21 & 167,95 & - & - \\
\hline $\mathrm{Ca} \%$ & - & 1,67 & 2,55 & 2,92 & 3,02 & 4,86 & 2,31 & 3,41 & 3,10 & 1,70 & - & - \\
\hline Ca-mg & - & 22,29 & 69,58 & 90,24 & 99,41 & 198,21 & 71,54 & 93,00 & 200,05 & 28,79 & - & - \\
\hline$M_{8} \%$ & - & 0,23 & 0,16 & 0,27 & 0,31 & 0,23 & 0,21 & 0,25 & 0,17 & 0,25 & - & - \\
\hline$M_{B}-m_{B}$ & - & 4,79 & 7,48 & 18,09 & 12,48 & 18,46 & 19,21 & 22,19 & 24,10 & 13,77 & - & - \\
\hline S\% & - & 0,33 & 0,17 & 0,15 & 0,18 & 0,11 & 0,21 & 0,25 & 0,17 & 0,25 & - & - \\
\hline
\end{tabular}

Sci. agric., Piracicaba, 50(2):193-203, jun./set., 1993 


\begin{tabular}{|c|c|c|c|c|c|c|c|c|c|c|c|c|}
\hline S-mg & - & 6,18 & 6,34 & 8,41 & 10,82 & 9,04 & 21,99 & 27,21 & 20,19 & 19,31 & - & - \\
\hline & \multicolumn{12}{|c|}{ L. NATAL } \\
\hline $\mathrm{N} \%$ & 2,35 & 2,46 & 2,48 & 2,55 & 2,43 & 2,98 & 2,77 & 2,21 & 1,91 & 2,63 & - & - \\
\hline $\mathrm{N} \cdot \mathrm{mg}$ & 35,82 & 64,87 & 132,53 & 125,49 & 156,33 & 178,25 & 202,16 & 247,87 & 244,86 & 215,81 & 262,99 & 181,32 \\
\hline $\mathbf{P F}$ & 0,11 & 0,12 & 0,16 & 0,13 & 0,11 & 0,10 & 0,02 & 0,12 & 0,10 & 0,08 & 0,09 & 0,97 \\
\hline P-mg & 3,55 & 7,72 & 14,42 & 15,58 & 17,43 & 19,07 & 2,64 & 30,85 & 23,68 & 20,08 & 24,57 & 17,58 \\
\hline $\mathrm{K} \%$ & 1,39 & 1,54 & 1,58 & 1,79 & 1,35 & 1,14 & 1,26 & 1,33 & 1,14 & 1,16 & 0,97 & $\cdot$ \\
\hline K-mg & 38,31 & 88,85 & 184,67 & 205,60 & 216,20 & 260,86 & 265,48 & 331,67 & 265,88 & 171.49 & 318,52 & 188,84 \\
\hline $\mathrm{Ca} \%$ & 4,64 & 3,05 & 2,58 & 3,71 & 3,76 & 4,56 & 3,74 & 3,43 & 3,68 & 4,51 & 2,77 & $\cdot$ \\
\hline Ca-mg & 14,82 & 41,91 & 62,90 & 61,59 & 126,93 & 223,72 & 67,69 & 123,85 & 174,63 & 42,75 & 167,77 & 75,51 \\
\hline $\mathrm{Mg}_{\mathrm{B}} \%$ & 0,37 & 0,23 & 0,19 & 0,26 & 0,19 & 0,12 & 0,14 & 0,19 & 0,24 & 0,17 & 0,16 & 0,16 \\
\hline$M_{B}-m_{B}$ & 5,58 & 6,56 & 10,70 & 13,72 & 19,24 & 9,85 & 14,35 & 23,14 & 24,19 & 17,21 & 23,11 & 15,74 \\
\hline s\% & 0,16 & 0,34 & 0,16 & 0,24 & 0,20 & 0,16 & 0,10 & 0,21 & 0,21 & 0,18 & 0,18 & 0,17 \\
\hline \multirow[t]{2}{*}{ s-mg } & 3,34 & 17,60 & 15,37 & 9,33 & 10,54 & 13,36 & 6,69 & 30,14 & 27,90 & 33,70 & 24,23 & 19,96 \\
\hline & \multicolumn{12}{|c|}{ L. PERA } \\
\hline$N \%$ & 1,93 & 2,38 & 2,90 & 2,34 & 2,09 & 2,32 & 2,52 & 2,46 & 2,47 & 2,36 & - & $\cdot$ \\
\hline $\mathrm{N}-\mathrm{mg}$ & 30,10 & 88,70 & 143,17 & 163,06 & 149,21 & 196,25 & 180,32 & 295,52 & 252,34 & 188,75 & 268,45 & - \\
\hline$P \%$ & 0,10 & 0,10 & 0,09 & 0,12 & 0,10 & 0,11 & 0,11 & 0,10 & 0,10 & 0,08 & 0,09 & $\cdot$ \\
\hline P-mg & 3,51 & 9,05 & 15,14 & 21,66 & 20,16 & 22,05 & 18,86 & 28,28 & 23,60 & 19,83 & 23,74 & - \\
\hline $\mathrm{K} \%$ & 1,41 & 1,41 & 1,30 & i, 74 & 1,58 & 1,14 & 0,78 & 1,18 & 1,07 & 1,18 & 1,20 & - \\
\hline K-mg & 36,26 & 99,53 & 177,20 & 238,16 & 240,06 & 268,04 & 183,98 & 297,52 & 270,74 & 211,03 & 307,59 & - \\
\hline $\mathrm{Ca} \%$ & 3,65 & 2,83 & 3,63 & 3,10 & 3,13 & 5,03 & 3,49 & 3,46 & 2,03 & 4,67 & 3,00 & $\cdot$ \\
\hline Ca-mg & 12,29 & 43,89 & 120,10 & 88,91 & 137,10 & 257,43 & 73,04 & 100,50 & 168,11 & 36,98 & 138,23 & - \\
\hline $\mathrm{Mg}_{\mathrm{g}} \%$ & 0,37 & 0,20 & 0,11 & 0,20 & 0,26 & 0,09 & 0,14 & 0,19 & 0,20 & 0,20 & 0,18 & - \\
\hline Mg-mg & 4,83 & 7,11 & 8,02 & 16,66 & 21,14 & 7,07 & 19,25 & 22,36 & 20,88 & 16,90 & 18,42 & - \\
\hline s\% & 0,11 & 0,36 & 0,09 & 0,15 & 0,18 & 0,13 & 0,22 & 0,20 & 0,20 & 0,15 & 0,18 & $\cdot$ \\
\hline \multirow[t]{2}{*}{ S-mg } & 3,49 & 23,72 & 5,26 & 7,24 & 13,94 & 9,66 & 15,11 & 33,00 & 22,94 & 19,47 & 2091 & - \\
\hline & \multicolumn{12}{|c|}{ L. VALENCIA } \\
\hline $\mathrm{N} \%$ & 2,24 & 2,30 & 2,71 & 2,22 & 2,28 & 2,50 & 3,00 & 2,78 & 2,71 & 2,69 & 2,79 & 2,95 \\
\hline $\mathrm{N}-\mathrm{mg}$ & 30,92 & 76,60 & 149,71 & 140,88 & 162,53 & 187,98 & 188,63 & 298,55 & 269,73 & 252,90 & 328,45 & 251,84 \\
\hline $\mathrm{P} \%$ & 0,09 & 0,10 & 0,08 & 0,10 & 0,10 & 0,11 & 0,11 & 0,13 & 0,09 & 0,12 & 0,12 & 0,09 \\
\hline P-mB & 3,15 & 7,78 & 13,65 & 17,64 & 23,21 & 21,44 & 17,75 & 32,00 & 30,28 & 20,25 & 27,18 & 24,48 \\
\hline K\% & 1,76 & 2,22 & 1,66 & 1,76 & 1,58 & 1,64 & 1,07 & 1,58 & 1,39 & 1,39 & 1,28 & 1,42 \\
\hline K-mg & 46,78 & 111,19 & 215,36 & 261,11 & 305,72 & 254,94 & 222,91 & 403,09 & 371,40 & 234,90 & 393,05 & 309,97 \\
\hline $\mathrm{Ca} \%$ & 3,93 & 1,73 & 1,15 & 2,94 & 3,24 & 3,93 & 2,75 & 2,99 & 3,52 & 1,95 & 4,31 & 2,59 \\
\hline Ca-mg & 10,61 & 48,88 & 64,50 & 73,90 & $t 53,83$ & 283,57 & 78,28 & 141,05 & 239,62 & 47,91 & 182,88 & 85,90 \\
\hline $\mathrm{M}_{\mathrm{B}} \%$ & 0,42 & 0,24 & 0,24 & 0,29 & 0,18 & 0,23 & 0,27 & 0,22 & 0,20 & 0,29 & 0,25 & 0,17 \\
\hline$M_{g-m g}$ & 5,10 & 8,13 & 12,75 & 17,80 & 18,45 & 12,54 & 18,19 & 28,22 & 28,27 & 21,60 & 28,47 & 20,93 \\
\hline S\% & 0,16 & 0,34 & 0,21 & 0,15 & 0,17 & 0,10 & 0,19 & 0,18 & 0,16 & 0,24 & 0,27 & 0,16 \\
\hline s-mg & 2,85 & 14,60 & 19,03 & 16,11 & 15,66 & 17,18 & 20,24 & 31,83 & 27,14 & 30,71 & 24,71 & 22,11 \\
\hline
\end{tabular}


TABELA 2 - Conoentraçio em ppon de micromutrientes nas folhas e extraçïo pelos frutos em funcî́o da idade.

\begin{tabular}{|c|c|c|c|c|c|c|c|c|c|c|c|c|}
\hline \multirow[t]{2}{*}{ Nutrientes } & \multicolumn{12}{|c|}{ Idade dos frutos em meses após florescimento } \\
\hline & 1 & 2 & 3 & 4 & 5 & 6 & 7 & 8 & 9 & 10 & 11 & 12 \\
\hline & \multicolumn{12}{|c|}{ T. CRAVO } \\
\hline B-ppm & 22 & 29 & 23 & 38 & 31 & 51 & 38 & 63 & - & - & - & - \\
\hline B- $\mu \mathrm{g}$ & 23 & 100 & 96 & 367 & 239 & 280 & 189 & 503 & - & - & - & - \\
\hline Cu-ppm & 29 & 43 & 68 & 46 & 45 & 47 & 50 & 75 & - & - & - & - \\
\hline $\mathrm{Cu}-\mu \mathrm{g}$ & 8 & 24 & 30 & 162 & 73 & 81 & 63 & 66 & - & - & - & - \\
\hline Fe-ppm & 107 & 64 & 73 & 104 & 135 & 116 & 124 & 180 & - & - & - & - \\
\hline $\mathrm{Fe}-\mu \mathrm{g}$ & 49 & 53 & 8 & 279 & 216 & 415 & 240 & 338 & - & - & - & - \\
\hline Mn-ppm & 34 & 20 & 19 & 20 & 35 & 43 & 33 & 50 & - & - & - & - \\
\hline $\mathrm{Mn}-\mu \mathrm{g}$ & 13 & 14 & 7 & 108 & 63 & 78 & 66 & 156 & - & - & - & - \\
\hline Zn-ppm & 48 & 32 & 36 & 34 & 47 & 44 & 40 & 49 & - & - & - & - \\
\hline \multirow[t]{2}{*}{$\mathrm{Zn}-\mu \mathrm{g}$} & 22 & 48 & 47 & 161 & 52 & 181 & 333 & 160 & - & - & - & - \\
\hline & \multicolumn{12}{|c|}{ L. HAMLIN } \\
\hline B-ppm & 28 & 20 & 36 & 36 & 29 & 46 & 37 & 38 & - & - & - & - \\
\hline B- $\mu \mathrm{g}$ & 67 & 139 & 165 & 180 & 271 & 351 & 335 & 490 & - & - & - & - \\
\hline Cu-ppm & 75 & 27 & 42 & 55 & 62 & 39 & 34 & 46 & - & - & - & - \\
\hline $\mathrm{Cu}-\mu \mathrm{g}$ & 13 & 15 & 10 & 43 & 67 & 75 & 83 & 83 & - & - & - & - \\
\hline Fe-ppm & 75 & 77 & 42 & 168 & 178 & 134 & 141 & 197 & - & - & - & - \\
\hline Fe- $\mu \mathrm{g}$ & 197 & 57 & 188 & 157 & 251 & 298 & 552 & 742 & - & - & - & - \\
\hline Mn-ppm & 37 & 23 & 33 & 30 & 39 & 40 & 36 & 45 & - & - & - & - \\
\hline $\mathrm{Mn}-\mu \mathrm{g}$ & 30 & 16 & 14 & 12 & 54 & 63 & 78 & 339 & - & - & - & - \\
\hline $\mathrm{Zn}$-ppm & 47 & 31 & 46 & 40 & 45 & 52 & 35 & 39 & - & - & - & - \\
\hline \multirow[t]{2}{*}{$\mathrm{Zn}-\mu \mathrm{g}$} & 31 & 78 & 64 & 99 & 150 & 161 & 224 & 380 & - & - & - & - \\
\hline & \multicolumn{12}{|c|}{ T. MURCOTT } \\
\hline B-ppm & 29 & 25 & 27 & 28 & 29 & 23 & 36 & 31 & 34 & 36 & - & - \\
\hline B- $\mu \mathrm{g}$ & 59 & 124 & 208 & 167 & 279 & 227 & 323 & 264 & 166 & 174 & - & - \\
\hline Cu-ppm & 163 & 116 & 103 & 156 & 159 & 122 & 154 & 121 & 105 & 128 & - & - \\
\hline $\mathrm{Cu}-\mu \mathrm{g}$ & 35 & 117 & 141 & 114 & 195 & 153 & 80 & 161 & 113 & 123 & - & - \\
\hline Fe-ppm & 51 & 67 & 107 & 182 & 116 & 120 & 123 & 169 & 138 & 115 & - & - \\
\hline Fe- $\mu \mathrm{g}$ & 25 & 30 & 327 & 201 & 554 & 500 & 627 & 410 & 225 & 300 & - & - \\
\hline Mn-ppm & 31 & 27 & 38 & 35 & 39 & 31 & 44 & 42 & 42 & 42 & - & - \\
\hline$M n-\mu g$ & 25 & 8 & 71 & 72 & 114 & 110 & 230 & 174 & 41 & 57 & - & - \\
\hline Zn-ppm & 67 & 60 & 47 & 43 & 57 & 52 & 43 & 41 & 66 & 44 & - & - \\
\hline
\end{tabular}




\begin{tabular}{|c|c|c|c|c|c|c|c|c|c|c|c|c|}
\hline $\mathrm{Zn}-\mu \mathrm{g}$ & 57 & 123 & 177 & 163 & 261 & 93 & 104 & 298 & 307 & 109 & - & - \\
\hline & \multicolumn{12}{|c|}{ L. NATAL } \\
\hline B-ppm & 27 & 21 & 32 & 28 & 36 & 20 & 28 & 45 & 50 & 27 & 26 & 30 \\
\hline B- $\mu \mathrm{g}$ & 62 & 147 & 218 & 197 & 261 & 346 & 432 & 624 & 427 & 386 & 316 & 243 \\
\hline Cu-ppm & 67 & 35 & 23 & 39 & 60 & 27 & 29 & 41 & 19 & 18 & 11 & 10 \\
\hline $\mathrm{Cu}-\mu \mathrm{g}$ & 17 & 21 & 12 & 81 & 105 & 124 & 83 & 95 & 83 & 66 & 72 & 34 \\
\hline Fe-ppm & 102 & 110 & 68 & 116 & 163 & 156 & 127 & 208 & 148 & 113 & 120 & 130 \\
\hline $\mathrm{Fe}-\mu \mathrm{g}$ & 64 & 128 & 60 & 211 & 318 & 391 & 484 & 961 & 414 & 374 & 415 & 442 \\
\hline Mn-ppm & 33 & 24 & 26 & 22 & 38 & 38 & 34 & 47 & 37 & 25 & 27 & 25 \\
\hline Mn- $\mu \mathrm{g}$ & 27 & 18 & 12 & 33 & 67 & 107 & 110 & 397 & 172 & 395 & 116 & 56 \\
\hline Zn-ppm & 25 & 24 & 22 & 23 & 30 & 55 & 48 & 55 & 32 & 47 & 39 & 29 \\
\hline \multirow[t]{2}{*}{$\mathrm{Zn}-\mu \mathrm{g}$} & 32 & 65 & 84 & 132 & 595 & 223 & 357 & 421 & 324 & 140 & 399 & 191 \\
\hline & \multicolumn{12}{|c|}{ L. PERA } \\
\hline B-ppm & 25 & 37 & 18 & 29 & 34 & 29 & 26 & 30 & 31 & 28 & 30 & - \\
\hline B- $\mu \mathrm{g}$ & 34 & 151 & 204 & 264 & 312 & 479 & 292 & 531 & 367 & 308 & 270 & - \\
\hline Cu-ppm & 7 & 6 & 8 & 10 & 16 & 12 & 10 & 11 & 14 & 27 & 15 & - \\
\hline $\mathrm{Cu}-\mu \mathrm{g}$ & 3 & 19 & 19 & 25 & 50 & 77 & 47 & 29 & 73 & 39 & 83 & - \\
\hline Fe-ppm & 102 & 94 & 156 & 157 & 171 & 143 & 133 & 154 & 136 & 112 & 140 & - \\
\hline $\mathrm{Fe}-\mu \mathrm{g}$ & 58 & 35 & 32 & 328 & 243 & 379 & 298 & 825 & 396 & 178 & 529 & - \\
\hline Mn-ppm & 43 & 34 & 29 & 29 & 40 & 27 & 24 & 34 & 35 & 24 & 43 & - \\
\hline$M n-\mu g$ & 34. & 71 & 87 & 163 & 108 & 315 & 98 & 324 & 224 & 84 & 160 & - \\
\hline Zn-ppm & 39 & 23 & 31 & 23 & 36 & 24 & 22 & 29 & 40 & 32 & 31 & - \\
\hline \multirow[t]{2}{*}{$\mathrm{Zn}-\mu \mathrm{g}$} & 34 & 71 & 87 & 163 & 108 & 314 & 98 & 324 & 224 & 184 & 160 & - \\
\hline & \multicolumn{12}{|c|}{ L. VALÊNCIA } \\
\hline B-ppm & 30 & 34 & 35 & 39 & 27 & 38 & 34 & 38 & 40 & 43 & 50 & 42 \\
\hline B-mg & 52 & 165 & 262 & 331 & 412 & 467 & 417 & 150 & 540 & 600 & 449 & 501 \\
\hline Cu-ppm & 156 & 78 & 151 & 291 & 163 & 118 & 159 & 170 & 159 & 107 & 47 & 48 \\
\hline $\mathrm{Cu}-\mu \mathrm{g}$ & 16 & 25 & 99 & 92 & 113 & 120 & 88 & 155 & 151 & 95 & 107 & 96 \\
\hline Fe-ppm & 91 & 132 & 195 & 197 & 193 & 137 & 142 & 175 & 143 & 137 & 169 & 154 \\
\hline $\mathrm{Fe}-\mu \mathrm{g}$ & 117 & 76 & 643 & 313 & 325 & 1104 & 313 & 834 & 382 & 442 & 412 & 401 \\
\hline Mn-ppm & 43 & 24 & 27 & 34 & 36 & 28 & 32 & 50 & 37 & 42 & 45 & 40 \\
\hline$M n-\mu g$ & 27 & 41 & 23 & 96 & 102 & 59 & 357 & 180 & 150 & 70 & 69 & 61 \\
\hline $\mathrm{Zn-ppm}$ & 24 & 19 & 19 & 24 & 17 & 20 & 19 & 41 & 35 & 34 & 42 & 35 \\
\hline $\mathrm{Zn}-\mu \mathrm{g}$ & 30 & 66 & 81 & 159 & 98 & 211 & 336 & 407 & 303 & 183 & 203 & 330 \\
\hline
\end{tabular}


Tabela 3 - Extração total de nutrientes por árvore dos cultivares.

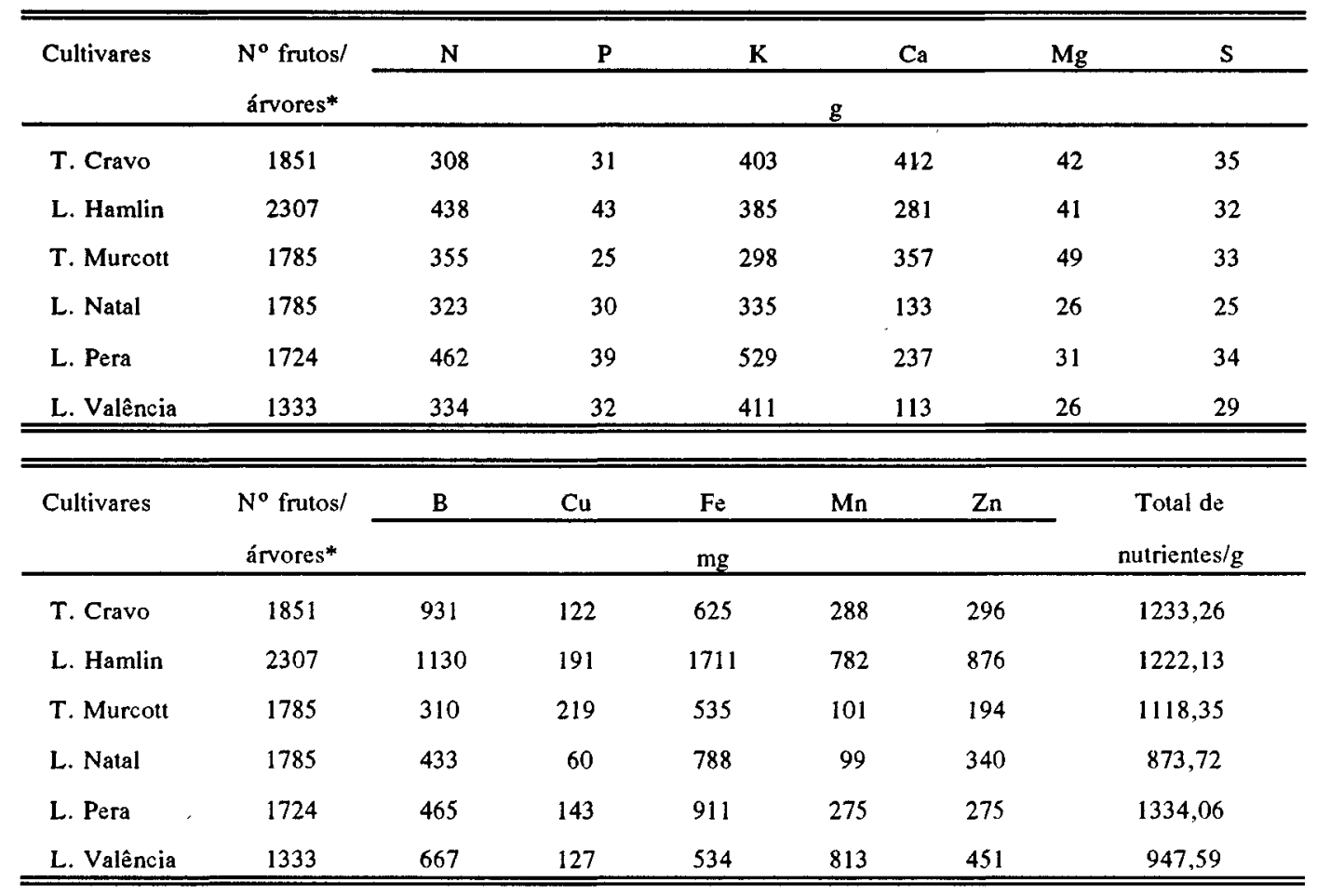

* FIGUEREDO (1991).

Dentre os micronutrientes, o $\mathrm{Fe}$ foi exportado em maiores quantidades seguido do $B$, $\mathrm{Zn}, \mathrm{Mn}$ e $\mathrm{Cu}$. A extração total de nutrientes, pelos frutos dos diversos cultivares mostra que a $\mathrm{L}$. Pera ocupa o primeiro lugar, seguida da T.Cravo, L. Hamlin, L. Valência. A L. Natal, exporta as menores quantidades de nutrientes apesar de acusar uma produção de frutos idêntica à $T$. Murcott.

Em frente a estes dados, as observações de LABANAUSKAS \& HANDY (1972), são corretas quando afirmaram a exportação de nutrientes pelos frutos dos citros é pequena, em confronto aos nutrientes que são aplicados ao solo.

Quantidades de suco produzido por fruto e concentração de ácido ascórbico.

Pelo exame da figura 2, observa-se que a cultivar $\mathbf{L}$.Valência produziu a maior quantidade de suco, cerca de $90 \mathrm{ml}$ por fruto. A menor produção, coube as cultivares T. Cravo e L. Hamlin. Houve uma redução na produção de suco nas épocas que antecederam a colheita devido a redução no peso da matéria seca dos frutos.

A figura 3 acusa a produção de ácido ascórbico em $\mathrm{mg} / 100 \mathrm{ml}$ de suco e observa-se que novamente a $L$. Valência produziu a maior quantidade de ácido ascórbico na ordem de $70 \mathrm{mg}$ por fruto, ocorrendo contudo um decréscimo com a idade do fruto.

Em segundo lugar na produção de ácido ascórbico, situa-se a L. Natal com uma produção máxima de $65 \mathrm{mg}$ de ácido ascórbico ao $7^{\circ}$ mês. A cultivar com a menor produção de ácido ascórbico foi a T. Murcott, chegando no $8^{\circ}$ mês a $37 \mathrm{mg}$. Os valores encontrados na literatura apontam entre 40 a $70 \mathrm{mg}$ de ácido ascórbico/100 $\mathrm{ml}$ de suco (ERICKSON, 1968).

Segundo BIRDSALL et al. (1961) e RODRIGUEZ (1991), o teor de ácido ascórbico decresce com a maturação das laranjas e tangerinas. Segundo ainda RODRIGUEZ (1991), nos frutos, somente um quarto do ácido ascórbico está no suco, ficando o restante na casca e no flavedo. 

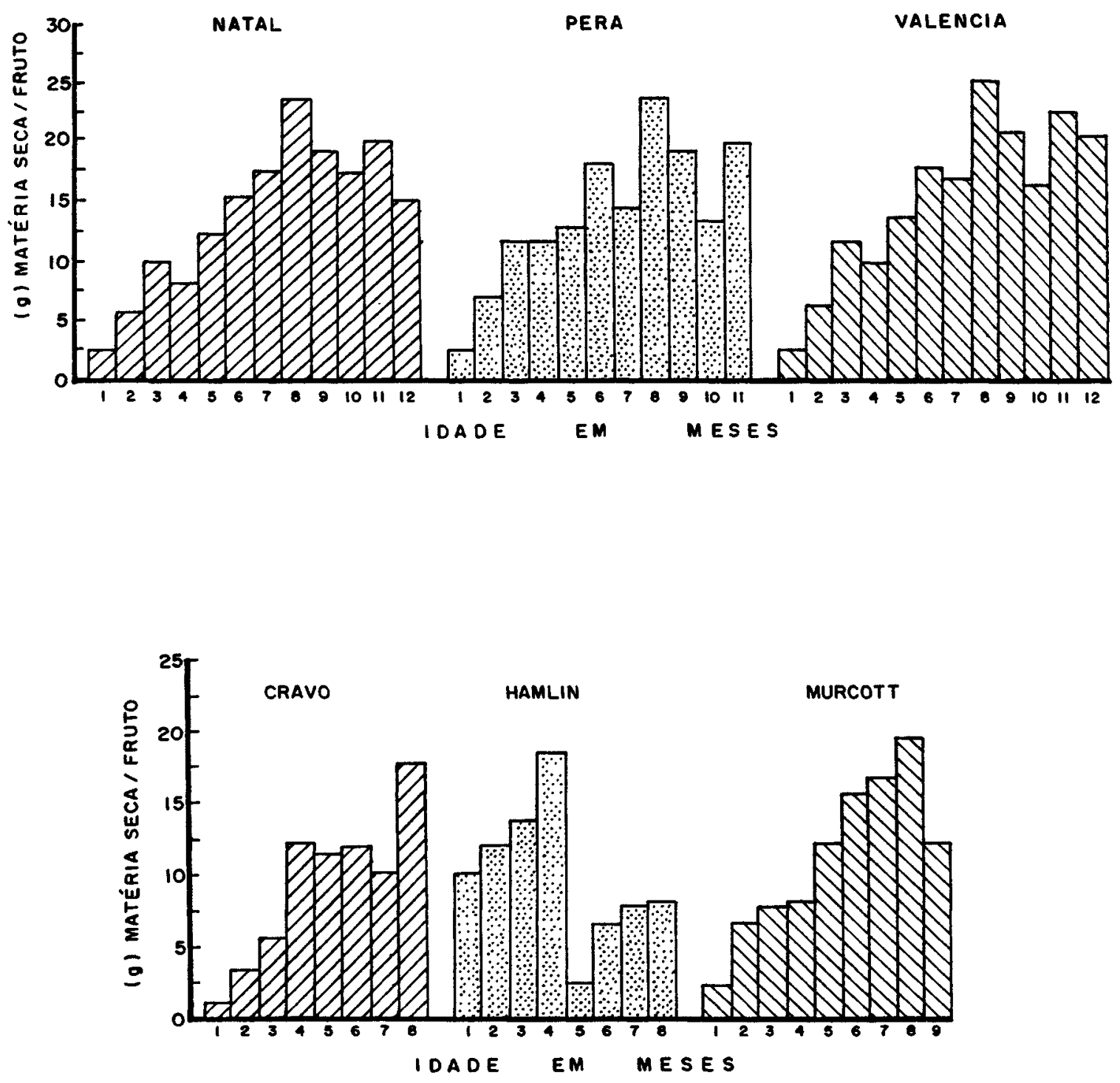

Figura 1 - Variação na produção de matéria seca por cultivar de citros, por idade dos frutos. 


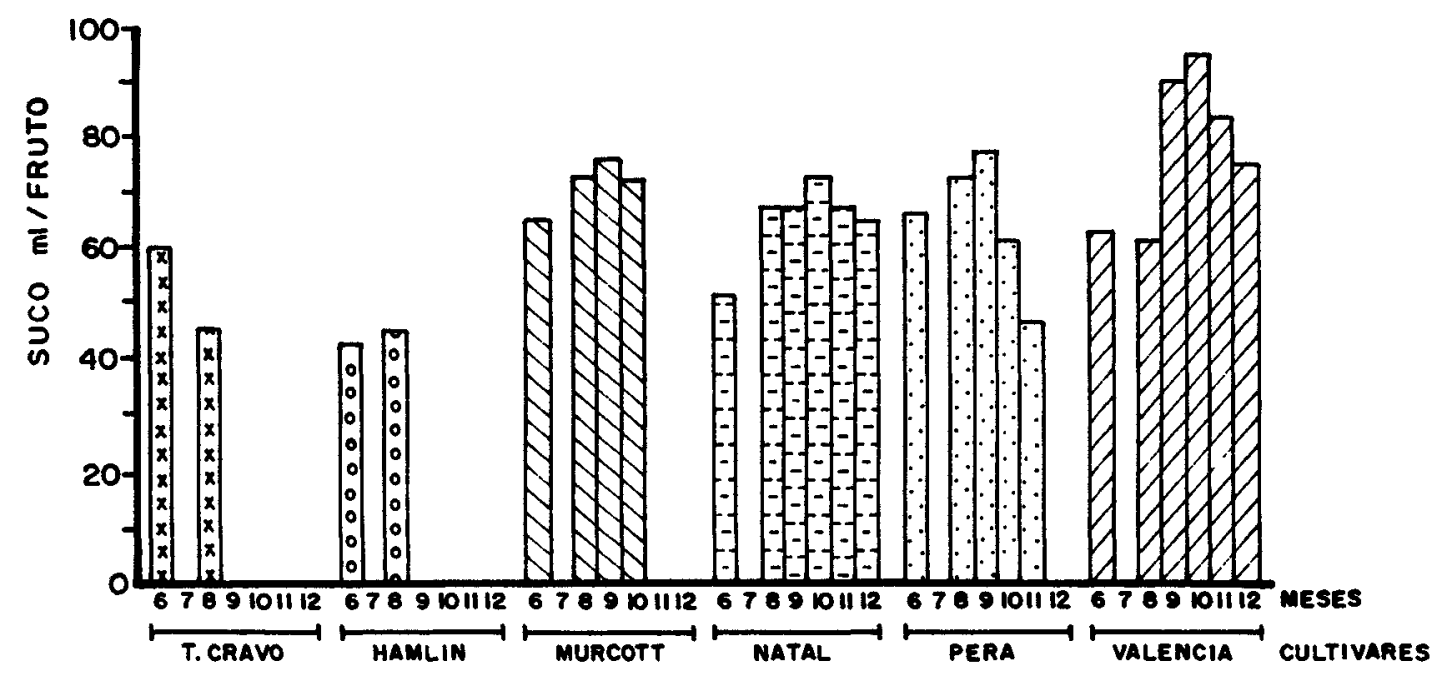

Figura 2 - Produção de suco (ml) por cultivar, em função da idade dos frutos.

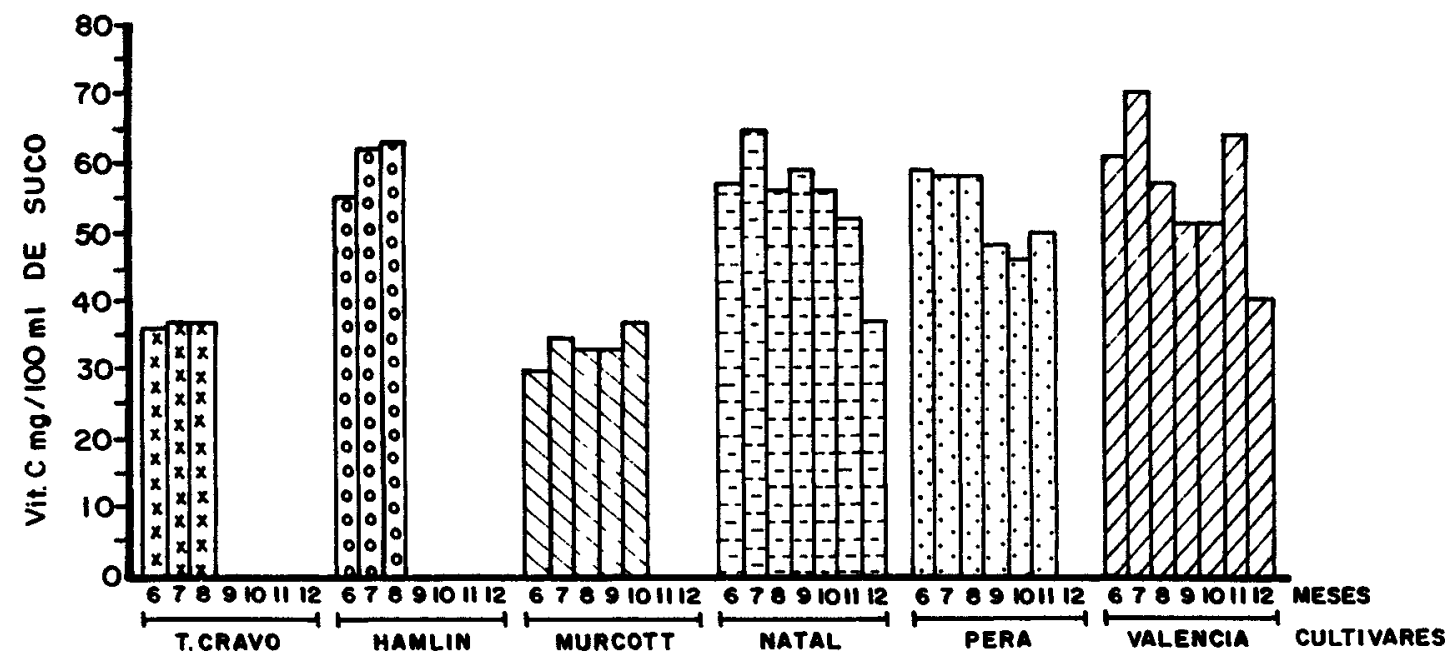

Figura 3 - Concentração de ácido ascórbico (mg/100 $\mathrm{ml}$ de suco) nos cultivares, em função da idade dos frutos.

Sci. agric., Piracicaba, 50(2):193-203, jun./set., 1993 


\section{CONCLUSÓES}

1. O aumento da matéria seca intensifica-se, a partir do segundo mes após o florescimento.

2. Com exceção da T. Cravo, ocorre diminuição na produção de matéria seca ao final do ciclo.

3. A concentração dos macro e micronutrientes nas folhas apresenta oscilações durante o desenvolvimento do fruto.

4. A ordem decrescente de extração de nutrientes foi: $\mathrm{K}, \mathrm{N}, \mathrm{Ca}, \mathrm{Mg}, \mathrm{P}=\mathrm{S}, \mathrm{Fe}, \mathrm{B}, \mathrm{Zn}, \mathrm{Mn}, \mathrm{Cu}$.

5. A capacidade de exportação de nutrientes pelos cultivares $\epsilon$, em ordem decrescente: $L$. Pera, $L$. Hamlin $=$ T. Cravo, T. Murcott, L. Valência, L. Natal.

6. A quantidade de suco produzido por fruto oscila entre 43 a $95 \mathrm{ml}$.

7. A concentração de ácido ascórbico em $\mathrm{mg} / 100$ ml, oscila entre 30 a 95.

\section{AGRADECIMENTOS}

Os autores agradecem o Eng ${ }^{\circ} \mathrm{Agr}^{\circ} \mathrm{Sr}$. José Renato Melare, Profissional da Fazenda Sete Lagoas Agrícola S.A.

\section{REFERENCIAS BIBLIOGRÁFICAS}

BATAGLIA, O.C.; RODRIGUEZ, O.; HIROCE, R; GALLO, J.R.; FURLANI, P.R.; FURLANI, A.M.C. Extração de nutrientes por frutos cítricos na collheita. In: CONGRESSO BRASILEIRO DE FRUTICULTURA 4., 1978. Salvador, Anais... Cruz das Almas: SBF. 1978. p.117-123.

BIRDSALL, J.J.; DERSE, P.H.; TEPLY, L.J. Nutrients in California lemons and oranges. II. Vitamin, mineral, and proximate composition. Journal of the American Dietetic Association. Chicago, v.38, p.555-559, 1961 .

BOSCHI, C. Contributo alla statica chimica - agraria della caltivazione del limone. In: REUTHER, W.; BATCELOR, L.D.; WEBER, H.J. (Ed.) The citrus industry, Berkeley: Univ. of California, 1968, v.2, p.32-57.

CHAPMAN, H.D. The mineral nutrition of citrus. In : REUTHER, W.; BATCHELOR, L.D.; WEBBER, H.J. (Ed.). The citrus industry. Berkeley: University of California, 1968. v.2, cap.3, p.127-289.
COLBY, Y.E. California oranges and lemons. In: REUTHER, W.; BATCELOR, L.D.; WEEBER, H.J. (Ed.) The citrus industry, Berkely: University of California, 1968, v.2, p.58-85.

ERICKSON, L.C. The general physiology of citrus. In: REUTHER, W.; BATCHELOR, L.D.; WEBER, H.H., (Ed.) The citrus industry, Berkely: University of California, 1968. v.2, p.86-126.

FIGUEREDO, J.O. Variedades copa de valor comercial. In: RODRIGUEZ, O; VIEGAS, F.; POMPEU, JR.; AMARO, A.A., (Ed.) Citricultura brasileira, 2.ed. Campinas: Fundação Cargill, 1991 vol.1, p.228-264.

JACOBS, M.B. The chemical analysis of foods and food products. 3.ed. Princeton: Van Nostrand, 1958. 970p.

LABANAUSKAS, C.K.; HANDY, M.F. Mineral nutrient removal by California Valéncias. Citrograph, Los Angeles, v.58, p.44-60, 1972.

MALAVOLTA, E.; SIVA, A.Q.; CESAR, M.J.A.; TEOFLO SOBRINHO, J.; POMPEU, JR., J. Variação de matéria seca e de macronutrientes nos frutos de cinco variedades de citros durante o seu crescimento. In: Congresse Brasileiro de Fruticultura 7., 1984, Florianópolis. Anais... Florianópolis: SBF, 1984. p.424-434.

MALAVOLTA, E.; SILVA, A.Q.; SILVA, H.; CESAR, M.J.A.; TEOFILO SOBRINHO, J.; POMPEU, J. JR. Acumulação de matéria seca e de micronutrientes nos frutos de cinco variedades de citros durante o seu crescimento. In: Congresso Brasileiro de Fruticultura 9., 1987, Campinas, Anais... Campinas:SBF, 1988. v.1, p.205-8.

RODRIGUEZ, O. Aspectos físiologicos, nutrição e adubação dos citros. In: RODRIGUEZ, O.; VIEGAS, F.; POMPEU, JR, J.; AMARO, A.A., (Ed.), Citricultura brasileira, 2.ed., Campinas: Fundaçāo Cargill, 1991. v.1, p.419-475.

SAMUELS, G. Abonos para café. Revista de Agricultura de Puerto Rico, San Juan, v.49, p.121$125,1957$.

SARRUGE, J.R.; HAAG, H.P. Andise química em plantas. Piracicaba, ESALQ, Depto. de Química, 1974. 56p.

SMITH, P. Leaf analysis of citrus. In: CHILDERS, N.F. (Ed.) TEMPERATE TO TROPICAL FRUTT NUTRITION. Rutgers, New Brunswick: The State University, 1961 . p.208-228.

Trabalho enviado para publicação em 30.01 .92 Trabalho aceito para publicação em 16.04 .93 